\title{
Extensions of inequalities for sector matrices
}

Song Lin ${ }^{1}$ and Xiaohui Fu'

"Correspondence: fxh6662@sina.com

'School of Mathematics and Statistics, Hainan Normal University, Haikou, P.R. China

\begin{abstract}
In this note, we first prove an inequality for sector matrices. This complements a result due to Kittaneh and Sakkijha (Linear Multilinear Algebra, 2018, https://doi.org/10.1080/03081087.2018.1441800) concerning accretive-dissipative matrices. And then we present two singular value inequalities for sector matrices which are similar to Yang and Lu's inequalities (J. Inequal. Appl. 2018:183, 2018).
\end{abstract}

MSC: 47A63; 15A45

Keywords: Sector matrices; Accretive-dissipative matrices; Numerical ranges; Schatten $p$-norms

\section{Introduction}

We denote by $\mathbb{M}_{n}(\mathbb{C})$ the set of $n \times n$ complex matrices. For $A \in \mathbb{M}_{n}(\mathbb{C})$, the conjugate transpose of $A$ is denoted by $A^{*}$, and the matrices $\mathfrak{R} A=\frac{1}{2}\left(A+A^{*}\right)$ and $\Im A=\frac{1}{2 i}\left(A-A^{*}\right)$ are called the real part and imaginary part of $A$, respectively (e.g., [1, p. 6] and [6, p. 7]). Recall that a norm $\|\cdot\|$ on $\mathbb{M}_{n}(\mathbb{C})$ is unitarily invariant if $\|U A V\|=\|A\|$ for any $A \in \mathbb{M}_{n}(\mathbb{C})$ and unitarily matrices $U, V \in \mathbb{M}_{n}(\mathbb{C})$. For $p \geq 1$, the Schatten $p$-norm of $A \in \mathbb{M}_{n}(\mathbb{C})$ is defined as $\|A\|_{p}=\left(\sum_{j=1}^{n} \sigma_{j}^{p}(A)\right)^{\frac{1}{p}}$. If the eigenvalues of a square matrix $A \in \mathbb{M}_{n}(\mathbb{C})$ are all real, then we denote $\lambda_{j}(A)$ the $j$ th largest eigenvalue of $A$. The singular values of a complex matrix $A \in \mathbb{M}_{n}$ are the eigenvalues of $|A|:=\left(A^{*} A\right)^{\frac{1}{2}}$, and we denote $\sigma_{j}(A):=\lambda_{j}(|A|)$ the $j$ th largest singular value of $A$. A positive semidefinite matrix $A$ will be expressed as $A \geq 0$. Likewise, we write $A>0$ to refer that $A$ is a positive definite matrix.

$A \in \mathbb{M}_{n}(\mathbb{C})$ is an accretive-dissipative matrix if $\Re A$ and $\Im A$ are both positive semidefinite. This class of matrices has recently been considered by Lin [9] and Lin and Zhou [11].

Let $H \in \mathbb{M}_{n}(\mathbb{C})$ be a Hermitian matrix and let $f$ be a real-valued function defined on an interval containing all the eigenvalues of $H$. Then $f(H)$ is well defined through spectral decomposition. $f$ is called matrix concave if $f(\alpha A+(1-\alpha) B) \geq \alpha f(A)+(1-\alpha) f(B)$ for any two Hermitian matrices $A, B \in \mathbb{M}_{n}(\mathbb{C})$ and all $\alpha \in[0,1]$.

The numerical range of $A \in \mathbb{M}_{n}(\mathbb{C})$ is defined by

$$
W(A)=\left\{x^{*} A x \mid x \in \mathbb{C}^{n}, x^{*} x=1\right\} .
$$

For $\alpha \in[0, \pi / 2)$, let

$$
S_{\alpha}=\{z \in \mathbb{C}|\Re z \geq 0,| \Im z \mid \leq(\Re z) \tan (\alpha)\}
$$

(c) The Author(s) 2019. This article is distributed under the terms of the Creative Commons Attribution 4.0 International License (http://creativecommons.org/licenses/by/4.0/), which permits unrestricted use, distribution, and reproduction in any medium, provided you give appropriate credit to the original author(s) and the source, provide a link to the Creative Commons license, and indicate if changes were made. 
be a sector region on the complex plane. A matrix whose numerical range is contained in a sector region $S_{\alpha}$ is called a sector matrix [10]. Recent research interest in this class of matrices starts with a resolution of a problem from numerical analysis [3]. Some research results on sector matrices can be found in $[3,7,8,10,13]$.

Kittaneh and Sakkijha [7] proved the following Schatten- $p$ norm inequalities.

Theorem 1.1 (see [7, Theorem 2.7]) Let $S, T \in \mathbb{M}_{n}(\mathbb{C})$ be accretive-dissipative. Then

$$
2^{\frac{-p}{2}}\left(\|S\|_{p}^{p}+\|T\|_{p}^{p}\right) \leq\|S+T\|_{p}^{p} \leq 2^{\frac{3 p}{2}-1}\left(\|S\|_{p}^{p}+\|T\|_{p}^{p}\right) \quad \text { for } p \geq 1
$$

Recently, Yang and Lu [12] gave a generalization of Theorem 1.1.

Theorem 1.2 (see [12, Theorem 2.3]) Let $A_{1}, \ldots, A_{n} \in \mathbb{M}_{n}(\mathbb{C})$ be accretive-dissipative.

Then

$$
2^{\frac{-p}{2}} \sum_{j=1}^{n}\left\|A_{j}\right\|_{p}^{p} \leq\left\|\sum_{j=1}^{n} A_{j}\right\|_{p}^{p} \leq \frac{\left(2 n^{2}\right)^{\frac{p}{2}}}{n} \sum_{j=1}^{n}\left\|A_{j}\right\|_{p}^{p} \quad \text { for } p \geq 1 .
$$

In [5], Garg and Aujla presented the following inequalities:

$$
\begin{aligned}
& \prod_{j=1}^{k} \sigma_{j}\left(|A+B|^{r}\right) \\
& \quad \leq \prod_{j=1}^{k} \sigma_{j}\left(I_{n}+|A|^{r}\right) \prod_{j=1}^{k} \sigma_{j}\left(I_{n}+|B|^{r}\right) \quad \text { for } 1 \leq k \leq n, 1 \leq r \leq 2 ; \\
& \prod_{j=1}^{k} \sigma_{j}\left(I_{n}+f(|A+B|)\right) \\
& \quad \leq \prod_{j=1}^{k} \sigma_{j}\left(I_{n}+f(|A|)\right) \prod_{j=1}^{k} \sigma_{j}\left(I_{n}+f(|B|)\right) \quad \text { for } 1 \leq k \leq n,
\end{aligned}
$$

where $A, B \in \mathbb{M}_{n}(\mathbb{C})$ and $f:[0, \infty) \rightarrow[0, \infty)$ is a matrix concave function.

If $A, B \geq 0, r=1$ and $f(X)=X$ for any $X \in \mathbb{M}_{n}(\mathbb{C})$ in (1) and (2), then

$$
\prod_{j=1}^{k} \sigma_{j}(A+B) \leq \prod_{j=1}^{k} \sigma_{j}\left(I_{n}+A\right) \prod_{j=1}^{k} \sigma_{j}\left(I_{n}+B\right) \quad \text { for } 1 \leq k \leq n
$$

and

$$
\prod_{j=1}^{k} \sigma_{j}\left(I_{n}+A+B\right) \leq \prod_{j=1}^{k} \sigma_{j}\left(I_{n}+A\right) \prod_{j=1}^{k} \sigma_{j}\left(I_{n}+B\right) \quad \text { for } 1 \leq k \leq n .
$$

Based on the above inequalities, Yang and Lu (see [12, Theorem 2.7]) gave the inequalities for sector matrices which removed the absolute values in (1) and (2) from the right sides as follows. 
Theorem 1.3 (see [12, Theorem 2.7]) Let $A, B \in \mathbb{M}_{n}(\mathbb{C})$ be such that $W(A), W(B) \subseteq S_{\alpha}$. Then, for $1 \leq k \leq n$,

$$
\prod_{j=1}^{k} \sigma_{j}(A+B) \leq \sec ^{2 k}(\alpha) \prod_{j=1}^{k} \sigma_{j}\left(I_{n}+A\right) \prod_{j=1}^{k} \sigma_{j}\left(I_{n}+B\right)
$$

and

$$
\prod_{j=1}^{k} \sigma_{j}\left(I_{n}+A+B\right) \leq \sec ^{2 k}(\alpha) \prod_{j=1}^{k} \sigma_{j}\left(I_{n}+A\right) \prod_{j=1}^{k} \sigma_{j}\left(I_{n}+B\right) .
$$

By Fan's dominance principle [1, p. 93], the theorem below follows from (4) and (5).

Theorem 1.4 (see [12, Corollary 2.8]) Let $A, B \in \mathbb{M}_{n}(\mathbb{C})$ be such that $W(A), W(B) \subseteq S_{\alpha}$. Then

$$
\|A+B\| \leq \sec ^{2}(\alpha)\left\|I_{n}+A\right\|\left\|I_{n}+B\right\|
$$

and

$$
\left\|I_{n}+A+B\right\| \leq \sec ^{2}(\alpha)\left\|I_{n}+A\right\|\left\|I_{n}+B\right\| .
$$

In this paper, we will extend Theorem 1.2 to sector matrices. Furthermore, we present some inequalities for sector matrices which are similar to the inequalities (4) and (5). However, in some cases, our results are stronger than (4) and (5), respectively.

\section{Main results}

We begin this section with some lemmas which are useful to establish our main results.

Lemma 2.1 (see $[1, \mathrm{p} .73])$ Let $A \in \mathbb{M}_{n}(\mathbb{C})$. Then

$$
\lambda_{j}(\Re A) \leq \sigma_{j}(A), \quad j=1,2, \ldots, n .
$$

Consequently,

$$
\|\Re A\| \leq\|A\| .
$$

Lemma 2.2 (see [13, Lemma 3.1]) Let $A \in \mathbb{M}_{n}(\mathbb{C})$ be such that $W(A) \subseteq S_{\alpha}$ for some $\alpha \in$ $[0, \pi / 2)$. Then

$$
\|A\| \leq \sec (\alpha)\|\Re A\| .
$$

Lemma 2.3 (see [2, (4)]) Let $A_{1}, \ldots, A_{n} \in \mathbb{M}_{n}(\mathbb{C})$ be positive semidefinite. Then, for $p \geq 1$,

$$
\sum_{j=1}^{n}\left\|A_{j}\right\|_{p}^{p} \leq\left\|\sum_{j=1}^{n} A_{j}\right\|_{p}^{p} \leq n^{p-1} \sum_{j=1}^{n}\left\|A_{j}\right\|_{p}^{p}
$$


Lemma 2.4 (see $\left[4\right.$, Theorem 4.1]) Let $A \in \mathbb{M}_{n}(\mathbb{C})$ be such that $W(A) \subseteq S_{\alpha}$. Then

$$
\sigma_{j}(A) \leq \sec ^{2}(\alpha) \lambda_{j}(\Re A), \quad j=1,2, \ldots, n .
$$

The above inequality implies that there exists a unitary matrix $U \in \mathbb{M}_{n}(\mathbb{C})$ such that

$$
|A| \leq \sec ^{2}(\alpha) U \Re A U^{*}
$$

Lemma 2.5 (see [1, Theorem III.5.6]) Let $A, B \in \mathbb{M}_{n}(\mathbb{C})$. Then there exist unitary matrices $U, V \in \mathbb{M}_{n}(\mathbb{C})$ such that

$$
|A+B| \leq U|A| U^{*}+V|B| V^{*}
$$

For the above preparation, we present the first main result which is an extension of Theorem 1.1.

Theorem 2.6 Let $A_{1}, \ldots, A_{n} \in \mathbb{M}_{n}(\mathbb{C})$ be sector matrices. Then

$$
\cos ^{p}(\alpha) \sum_{j=1}^{n}\left\|A_{j}\right\|_{p}^{p} \leq\left\|\sum_{j=1}^{n} A_{j}\right\|_{p}^{p} \leq \sec ^{p}(\alpha) n^{p-1} \sum_{j=1}^{n}\left\|A_{j}\right\|_{p}^{p} \quad \text { for } p \geq 1 .
$$

Proof Let $A_{j}=B_{j}+i C_{j}$ be the Cartesian decompositions of $A_{j}, j=1, \ldots, n$. Then we have

$$
\begin{aligned}
\left\|\sum_{j=1}^{n} A_{j}\right\|_{p}^{p} & =\left\|\sum_{j=1}^{n}\left(B_{j}+i C_{j}\right)\right\|_{p}^{p} \\
& =\left\|\sum_{j=1}^{n} B_{j}+i \sum_{j=1}^{n} C_{j}\right\|_{p}^{p} \\
& \geq\left\|\sum_{j=1}^{n} B_{j}\right\|_{p}^{p} \quad(\text { by (9)) } \\
& \geq \sum_{j=1}^{n}\left\|B_{j}\right\|_{p}^{p} \quad(\text { by (11)) } \\
& \geq \cos ^{p}(\alpha) \sum_{j=1}^{n}\left\|A_{j}\right\|_{p}^{p} \quad \text { (by (10)) }
\end{aligned}
$$

which proves the first inequality.

To prove the second inequality, compute

$$
\begin{aligned}
\left\|\sum_{j=1}^{n} A_{j}\right\|_{p}^{p} & =\left\|\sum_{j=1}^{n}\left(B_{j}+i C_{j}\right)\right\|_{p}^{p} \\
& =\left\|\sum_{j=1}^{n} B_{j}+i \sum_{j=1}^{n} C_{j}\right\|_{p}^{p} \\
& \leq \sec ^{p}(\alpha)\left\|\sum_{j=1}^{n} B_{j}\right\|_{p}^{p} \text { (by (10)) }
\end{aligned}
$$




$$
\begin{aligned}
& \leq \sec ^{p}(\alpha) n^{p-1} \sum_{j=1}^{n}\left\|B_{j}\right\|_{p}^{p} \quad \text { (by (11)) } \\
& \leq \sec ^{p}(\alpha) n^{p-1} \sum_{j=1}^{n}\left\|A_{j}\right\|_{p}^{p} \quad \text { (by (9)), }
\end{aligned}
$$

which completes the proof.

Remark 2.7 Theorem 1.2 is immediate by setting $\alpha=\frac{\pi}{4}$ in (14). Moreover, when $n=2$ and $\alpha=\frac{\pi}{4}$ in Theorem 2.6, our result is Theorem 1.1.

Next, we end this section with a generalization of singular value inequality for two positive semidefinite matrices to sector matrices.

Theorem 2.8 Let $A, B \in \mathbb{M}_{n}(\mathbb{C})$ be such that $W(A), W(B) \subseteq S_{\alpha}$. Then, for $1 \leq k \leq n$, the following assertions hold:

$$
\prod_{j=1}^{k} \sigma_{j}(A+B) \leq \prod_{j=1}^{k} \sigma_{j}\left(I_{n}+\sec ^{2}(\alpha) A\right) \prod_{j=1}^{k} \sigma_{j}\left(I_{n}+\sec ^{2}(\alpha) B\right)
$$

and

$$
\prod_{j=1}^{k} \sigma_{j}\left(I_{n}+A+B\right) \leq \prod_{j=1}^{k} \sigma_{j}\left(I_{n}+\sec ^{2}(\alpha) A\right) \prod_{j=1}^{k} \sigma_{j}\left(I_{n}+\sec ^{2}(\alpha) B\right) .
$$

Proof Compute

$$
\begin{aligned}
\prod_{j=1}^{k} \sigma_{j}(A+B) & \leq \prod_{j=1}^{k} \sigma_{j}\left(I_{n}+|A|\right) \prod_{j=1}^{k} \sigma_{j}\left(I_{n}+|B|\right) \quad(\text { by }(1)) \\
& \leq \prod_{j=1}^{k} \sigma_{j}\left(I_{n}+\sec ^{2}(\alpha) U \Re A U^{*}\right) \prod_{j=1}^{k} \sigma_{j}\left(I_{n}+\sec ^{2}(\alpha) V \Re B V^{*}\right)
\end{aligned}
$$

(by (12))

$$
\leq \prod_{j=1}^{k} \sigma_{j}\left(I_{n}+\sec ^{2}(\alpha) A\right) \prod_{j=1}^{k} \sigma_{j}\left(I_{n}+\sec ^{2}(\alpha) B\right) \quad(\text { by }(8)),
$$

where $U, V$ are unitary matrices.

To prove (16), compute

$$
\begin{aligned}
\prod_{j=1}^{k} \sigma_{j}\left(\left|I_{n}+A+B\right|\right) & \leq \prod_{j=1}^{k} \sigma_{j}\left(U_{1}\left|I_{n}\right| U_{1}^{*}+V_{1}|A+B| V_{1}^{*}\right) \quad(\text { by }(13)) \\
& =\prod_{j=1}^{k} \sigma_{j}\left(I_{n}+|A+B|\right) \\
& \leq \prod_{j=1}^{k} \sigma_{j}\left(I_{n}+U_{2}|A| U_{2}^{*}+V_{2}|B| V_{2}^{*}\right) \quad(\text { by (13) })
\end{aligned}
$$




$$
\begin{aligned}
\leq & \prod_{j=1}^{k} \sigma_{j}\left(I_{n}+\sec ^{2}(\alpha) U_{3} \Re A U_{3}^{*}+\sec ^{2}(\alpha) V_{3} \Re B V_{3}^{*}\right) \\
& (\text { by }(12)) \\
\leq & \prod_{j=1}^{k} \sigma_{j}\left(I_{n}+\sec ^{2}(\alpha) \Re A\right) \prod_{j=1}^{k} s_{j}\left(I_{n}+\sec ^{2}(\alpha) \Re B\right) \\
& (\text { by }(3)) \\
\leq & \prod_{j=1}^{k} \sigma_{j}\left(I_{n}+\sec ^{2}(\alpha) A\right) \prod_{j=1}^{k} \sigma_{j}\left(I_{n}+\sec ^{2}(\alpha) B\right), \\
& (\text { by }(8)),
\end{aligned}
$$

where $U_{j}$ and $V_{j}, j=1,2,3$, are unitary matrices.

Thus

$$
\prod_{j=1}^{k} \sigma_{j}\left(I_{n}+A+B\right) \leq \prod_{j=1}^{k} \sigma_{j}\left(I_{n}+\sec ^{2}(\alpha) A\right) \prod_{j=1}^{k} \sigma_{j}\left(I_{n}+\sec ^{2}(\alpha) B\right) .
$$

This completes the proof.

The following examples show that neither (4) nor (15) is uniformly better than the other.

Example 2.9 Let

$$
A=e^{-\frac{\pi i}{4}}\left(\begin{array}{cc}
\frac{1}{8} & 0 \\
0 & \frac{1}{8}
\end{array}\right)=\left(\begin{array}{cc}
\frac{\sqrt{2}}{16}-\frac{\sqrt{2}}{16} i & 0 \\
0 & \frac{\sqrt{2}}{16}-\frac{\sqrt{2}}{16} i
\end{array}\right)
$$

and

$$
B=e^{-\frac{\pi i}{4}}\left(\begin{array}{cc}
\frac{1}{16} & 0 \\
0 & \frac{1}{16}
\end{array}\right)=\left(\begin{array}{cc}
\frac{\sqrt{2}}{32}-\frac{\sqrt{2}}{32} i & 0 \\
0 & \frac{\sqrt{2}}{32}-\frac{\sqrt{2}}{32} i
\end{array}\right)
$$

be such that $W(A), W(B) \subseteq S_{\frac{\pi}{4}}$.

For the right side of the inequality (4),

$$
\sec ^{4}(\alpha) \prod_{j=1}^{2} \sigma_{j}\left(I_{2}+A\right) \prod_{j=1}^{2} \sigma_{j}\left(I_{2}+B\right)=5.2098
$$

When $k=2$, for the right side of the inequality (15),

$$
\prod_{j=1}^{2} \sigma_{j}\left(I_{2}+\sec ^{2}(\alpha) A\right) \prod_{j=1}^{2} \sigma_{j}\left(I_{2}+\sec ^{2}(\alpha) B\right)=1.6886 .
$$

This shows that (15) is stronger than (4). 
Example 2.10 If

$$
A=e^{-\frac{\pi i}{4}}\left(\begin{array}{cc}
1+\frac{1}{2} i & 0 \\
0 & 1+\frac{1}{2} i
\end{array}\right)=\left(\begin{array}{cc}
\frac{3 \sqrt{2}}{4}-\frac{\sqrt{2}}{4} i & 0 \\
0 & \frac{3 \sqrt{2}}{4}-\frac{\sqrt{2}}{4} i
\end{array}\right)
$$

and

$$
B=e^{-\frac{\pi i}{4}}\left(\begin{array}{cc}
1+\frac{1}{3} i & 0 \\
0 & 1+\frac{1}{3} i
\end{array}\right)=\left(\begin{array}{cc}
\frac{2 \sqrt{2}}{3}-\frac{\sqrt{2}}{3} i & 0 \\
0 & \frac{2 \sqrt{2}}{3}-\frac{\sqrt{2}}{3} i
\end{array}\right)
$$

are such that $W(A), W(B) \subseteq S_{\frac{\pi}{4}}$, we also suppose $k=2$.

For the right side of the inequality (4),

$$
\sec ^{4}(\alpha) \prod_{j=1}^{2} \sigma_{j}\left(I_{2}+A\right) \prod_{j=1}^{2} \sigma_{j}\left(I_{2}+B\right)=69.8872 .
$$

For the right side of the inequality (15),

$$
\prod_{j=1}^{2} \sigma_{j}\left(I_{2}+\sec ^{2}(\alpha) A\right) \prod_{j=1}^{2} \sigma_{j}\left(I_{2}+\sec ^{2}(\alpha) B\right)=94.3901 .
$$

The example implies that the bound in (15) is weaker than that in (4).

Remark 2.11 Actually, the above examples also show that the inequalities (5) and (16) are not comparable.

Corollary 2.12 Let $A, B \in \mathbb{M}_{n}(\mathbb{C})$ be such that $W(A), W(B) \subseteq S_{\alpha}$. Then, for all unitarily invariant norms $\|\cdot\|$ on $\mathbb{M}_{n}(\mathbb{C})$,

$$
\|A+B\| \leq\left\|I_{n}+\sec ^{2}(\alpha) A\right\|\left\|I_{n}+\sec ^{2}(\alpha) B\right\|
$$

and

$$
\left\|I_{n}+A+B\right\| \leq\left\|I_{n}+\sec ^{2}(\alpha) A\right\|\left\|I_{n}+\sec ^{2}(\alpha) B\right\| .
$$

Proof From (15) and (16), we obtain for $1 \leq k \leq n$

$$
\prod_{j=1}^{k} \sigma_{j}^{\frac{1}{2}}(A+B) \leq \prod_{j=1}^{k} \sigma_{j}^{\frac{1}{2}}\left(I_{n}+\sec ^{2}(\alpha) A\right) \sigma_{j}^{\frac{1}{2}}\left(I_{n}+\sec ^{2}(\alpha) B\right)
$$

and

$$
\prod_{j=1}^{k} \sigma_{j}^{\frac{1}{2}}\left(I_{n}+A+B\right) \leq \prod_{j=1}^{k} \sigma_{j}^{\frac{1}{2}}\left(I_{n}+\sec ^{2}(\alpha) A\right) \sigma_{j}^{\frac{1}{2}}\left(I_{n}+\sec ^{2}(\alpha) B\right) .
$$


By the property that weak log-majorization implies weak majorization, we have for $1 \leq$ $k \leq n$

$$
\sum_{j=1}^{k} \sigma_{j}^{\frac{1}{2}}(A+B) \leq \sum_{j=1}^{k} \sigma_{j}^{\frac{1}{2}}\left(I_{n}+\sec ^{2}(\alpha) A\right) \sigma_{j}^{\frac{1}{2}}\left(I_{n}+\sec ^{2}(\alpha) B\right)
$$

and

$$
\sum_{j=1}^{k} \sigma_{j}^{\frac{1}{2}}\left(I_{n}+A+B\right) \leq \sum_{j=1}^{k} \sigma_{j}^{\frac{1}{2}}\left(I_{n}+\sec ^{2}(\alpha) A\right) \sigma_{j}^{\frac{1}{2}}\left(I_{n}+\sec ^{2}(\alpha) B\right) .
$$

Then, by the Cauchy-Schwarz inequality, for $1 \leq k \leq n$

$$
\sum_{j=1}^{k} \sigma_{j}^{\frac{1}{2}}(A+B) \leq\left(\sum_{j=1}^{k} \sigma_{j}\left(I_{n}+\sec ^{2}(\alpha) A\right)\right)^{\frac{1}{2}}\left(\sum_{j=1}^{k} \sigma_{j}\left(I_{n}+\sec ^{2}(\alpha) B\right)\right)^{\frac{1}{2}}
$$

and

$$
\sum_{j=1}^{k} \sigma_{j}^{\frac{1}{2}}\left(I_{n}+A+B\right) \leq\left(\sum_{j=1}^{k} \sigma_{j}\left(I_{n}+\sec ^{2}(\alpha) A\right)\right)^{\frac{1}{2}}\left(\sum_{j=1}^{k} \sigma_{j}\left(I_{n}+\sec ^{2}(\alpha) B\right)\right)^{\frac{1}{2}}
$$

By Fan's dominance principle [1, p. 93], we have

$$
\left\||A+B|^{\frac{1}{2}}\right\|^{2} \leq\left\|I_{n}+\sec ^{2}(\alpha) A\right\|\left\|I_{n}+\sec ^{2}(\alpha) B\right\|
$$

and

$$
\left\|\left|I_{n}+A+B\right|^{\frac{1}{2}}\right\|^{2} \leq\left\|I_{n}+\sec ^{2}(\alpha) A\right\|\left\|I_{n}+\sec ^{2}(\alpha) B\right\| .
$$

Let $A+B=U|A+B|, I_{n}+A+B=V\left|I_{n}+A+B\right|$ be the polar decomposition of $A+B$ and $I_{n}+A+B$, respectively, where $U$ and $V$ are unitary matrices. Thus, by (19), we have

$$
\begin{aligned}
\||A+B|\| & =\|U|A+B|\| \\
& =\left\|\left(|A+B|^{\frac{1}{2}}\right)^{2}\right\| \\
& \leq\left\||A+B|^{\frac{1}{2}}\right\|^{2} \\
& \leq\left\|I_{n}+\sec ^{2}(\alpha) A\right\|\left\|I_{n}+\sec ^{2}(\alpha) B\right\| .
\end{aligned}
$$

Similarly, by (20) we have

$$
\left\|I_{n}+A+B\right\| \leq\left\|I_{n}+\sec ^{2}(\alpha) A\right\|\left\|I_{n}+\sec ^{2}(\alpha) B\right\|,
$$

which completes the proof.

Remark 2.13 By computing Examples 2.9 and 2.10, it should be noticed here that neither (6) nor (17) is uniformly better than the other. When comparing the inequality (7) with (18), the same conclusion can be drawn. 
Taking $k=n$ in Theorem 2.8, we get the following corollary.

Corollary 2.14 Let $A, B \in \mathbb{M}_{n}(\mathbb{C})$ be such that $W(A), W(B) \subseteq S_{\alpha}$. Then

$$
|\operatorname{det}(A+B)| \leq\left|\operatorname{det}\left(I_{n}+\sec ^{2}(\alpha) A\right)\right|\left|\operatorname{det}\left(I_{n}+\sec ^{2}(\alpha) B\right)\right|
$$

and

$$
\left|\operatorname{det}\left(I_{n}+A+B\right)\right| \leq\left|\operatorname{det}\left(I_{n}+\sec ^{2}(\alpha) A\right)\right|\left|\operatorname{det}\left(I_{n}+\sec ^{2}(\alpha) B\right)\right| .
$$

\section{Acknowledgements}

Not applicable.

\section{Funding}

This paper is supported by Foundation for Scientific Research by the Ministry of Education of Hainan, China

(No. Hnky2019ZD-13) and Excellent Young Teachers Growth Program of Hainan Normal University, Haikou, China.

\section{Availability of data and materials}

Not applicable.

\section{Competing interests}

The authors declare that they have no competing interests.

\section{Authors' contributions}

All authors contributed almost the same amount of work to the manuscript. All authors read and approved the final manuscript.

\section{Publisher's Note}

Springer Nature remains neutral with regard to jurisdictional claims in published maps and institutional affiliations.

Received: 1 February 2019 Accepted: 15 April 2019 Published online: 02 May 2019

\section{References}

1. Bhatia, R.: Matrix Analysis. Springer, New York (1997)

2. Bhatia, R., Holbrook, J.A.R.: On the Clarkson-McCarthy inequalities. Math. Ann. 281, 7-12 (1988)

3. Drury, S.W.: Fischer determinantal inequalities and Higham's conjecture. Linear Algebra Appl. 439, 3129-3133 (2013)

4. Drury, S.W., Lin, M.: Singular value inequalities for matrices with numerical ranges in a sector. Oper. Matrices 8 , 1143-1148 (2014)

5. Garg, I., Aujla, J.: Some singular value inequalities. Linear Multilinear Algebra 66, 776-784 (2018)

6. Horn, R.A., Johnson, C.R.: Matrix Analysis, 2nd edn. Cambridge University Press, Cambridge (2013)

7. Kittaneh, F., Sakkijha, M.: Inequalities for accretive-dissipative matrices. Linear Multilinear Algebra (2018, in press). https://doi.org/10.1080/03081087.2018.1441800

8. Li, C.-K., Sze, N.: Determinantal and eigenvalue inequalities for matrices with numerical ranges in a sector. J. Math. Anal. Appl. 410, 487-491 (2014)

9. Lin, M.: Fischer type determinantal inequalities for accretive-dissipative matrices. Linear Algebra Appl. 438, 2808-2812 (2013)

10. Lin, M.: Some inequalities for sector matrices. Oper. Matrices 10, 915-921 (2016)

11. Lin, M., Zhou, D.: Norm inequalities for accretive-dissipative operator matrices. J. Math. Anal. Appl. 407, 436-442 (2013)

12. Yang, C., Lu, F.: Some generalizations of inequalities for sector matrices. J. Inequal. Appl. 2018, 183 (2018). https://doi.org/10.1186/s13660-018-1786-8

13. Zhang, F.: A matrix decomposition and its applications. Linear Multilinear Algebra 63, 2033-2042 (2015) 